ISSN 09753303

ACCOUNT Mis, vol.1, No. 2, Dec. 2002 - April 2003 pp.15-21

https:/ / doi.org/ 10.12725/ mjs.2.4

\title{
Application of Ruscheweyh Derivative Operator on Meromorphic Functions in the Unit Disk
}

K.S. Padmanabhan* and Rajalakshmi Rajagopalt

\begin{abstract}
Keywords and Phrases: Ruschweyh derivative, Differential inequalities, Hadamard product. ${ }^{\ddagger}$

In this paper we introduce a certain convolution operator $D^{\alpha+p-1}$ on meromorphic functions of the form

$f(z)=\frac{1}{z^{p}}+\sum a_{n} z^{n}$ in $0<|z|<1$

and deal with the application of the above operator to certain differential inequalities involving $D^{\alpha+p-1} f$.
\end{abstract}

\section{Introduction}

In a recent paper' Lin Jinlin has introduced a differential operator defined by Convolution on functions of the form

$f(z)=z^{p}+a_{p+1} z^{p+1}+\ldots$ (p a positive integer)

* Director (Retired), Ramanujan Institute for Advanced Study of Mathematics, University of Madras, India.

+ Department of Mathematics, Loyola College, Chennai-600 034, India. 
analytic in $|z|<1$ and, studied and obtained differential inequalities. In this paper we introduce a similar operator on functions $f \in M(p)$ where $M(p)$, p a positive integer denotes the class of meromorphic functions $f$ of the form

$$
f(z)=\frac{1}{z^{p}}+\frac{b_{1}}{z^{p-1}}+\ldots+\frac{b_{p-1}}{z}+\sum_{0}^{\infty} a_{n} z^{n}
$$

which are analytic in the punctured disc $E_{0}=\{z: 0<|z|<1\}$. We denote $f^{*} g$ the convolution (or Hadamard product of $f, g \in M(p)$ ) defined as follows. Let

$$
\begin{aligned}
& f(z)=\frac{1}{z^{p}}+\frac{b_{1}}{z^{p-1}}+\frac{b_{2}}{z^{p-2}} \ldots+\frac{b_{p-1}}{z}+\sum_{0}^{\infty} a_{n} z^{n} \\
& g(z)=\frac{1}{z^{p}}+\frac{c_{1}}{z^{p-1}}+\frac{c_{2}}{z^{p-2}} \ldots+\frac{c_{p-1}}{z}+\sum_{0}^{\infty} d_{n} z^{n}
\end{aligned}
$$

then

$$
\left(f^{*} g\right)(z)=\frac{1}{z^{p}}+\sum_{n=1}^{p-1} \frac{b_{n} c_{n}}{z^{p-n}}+\sum_{n=0}^{\infty} a_{n} d_{n} z^{n}
$$

Using the above convolution, we define the operator $D^{\alpha+p-1}$ on $f$ by

$$
D^{\alpha+p-1} f(z)=\frac{1}{z^{p}(1-z)^{\alpha+p}} * f(z)
$$

where $f(z) \in M(p)$ and $\alpha$ is any real number $>-p$.

For $\alpha=-(p-1)$, we get

$$
D^{0} f(z)=\frac{1}{z^{p}(1-z)} * f(z)=f(z)
$$

so that $D^{0}$ acts as the identity operator on $f$. 
We can easily prove that

$$
D^{\alpha+p-1} f(z)=\frac{1}{z^{p}} \frac{\left(z^{\alpha+2 p-1} f(z)\right)^{(\alpha+p-1)}}{(\alpha+p-1) !}
$$

If $\alpha$ is an integer not less than $-p+1$.

Therefore

$$
\begin{aligned}
D^{\alpha+p-1} f(z) & =\frac{1}{z^{p}(1-z)^{\alpha+p}} * f(z) \\
& =\frac{1}{z^{p}} \frac{\left(z^{\alpha+2 p-1} f(z)\right)^{(\alpha+p-1)}}{(\alpha+p-1) !}
\end{aligned}
$$

We propose to study the application of this Convolution operator to certain differential inequalities.

Now we need the following.

\section{Definition [1]}

Let $H(\alpha)$ be the set of complex valued functions; $h(r, s, t) ; h(r, s, t) ; C^{3} \rightarrow C$ (C is the complex plane) such that

i) $\quad h(r, s, t)$ is continuous in a domain $D \subset C^{3}$;

ii) $(1,1,1) \in D \quad$ and $\quad|h(1,1,1)|<J(J>1)$;

iii) $\quad h\left(J e^{i \theta}, \frac{(1+m)+(\alpha+p) J e^{i \theta}}{\alpha+p+1}, \frac{1}{\alpha+p+2} \times\right.$

$\left.\cdot\left[2+m+(\alpha+p) J e^{i \theta}+\frac{m-m^{2}+(\alpha+p) m J e^{i \theta}+L}{1+m+(\alpha+p) J e^{i \theta}}\right]\right) \mid>J$

whenever 


$$
\begin{aligned}
& \left(J e^{i \theta}, \frac{(1+m)+(\alpha+p) J e^{i \theta}}{\alpha+p+1}, \frac{1}{\alpha+p+2} \times\right. \\
& \left.\left[2+m+(\alpha+p) J e^{i \theta}+\frac{m-m^{2}+(\alpha+p) m J e^{i \theta}+L}{1+m+(\alpha+p) J e^{i \theta}}\right]\right) \in D
\end{aligned}
$$

with $\operatorname{Re} \leq m(m-1)$ for real $\theta$ and for real $m \geq \frac{J-1}{J+1}$.

Main Result

First we state the following lemma due to Miller and Mocanu², which we use in the sequel.

\section{Lemma}

Let $W(z)=a+W_{k} z^{k}+\ldots$ be regular in the unit disc $E$ with $W(z) \equiv a$ and $k \geq 1$.

If $z_{0}=r_{0} e^{i \theta}\left(0<r_{0}<1\right) \quad$ and $\left|W\left(z_{0}\right)\right|=\max _{|z| \leq r_{0}}|W(z)|$, then

i) $\quad z_{0} \frac{W^{\prime}\left(z_{0}\right)}{W\left(z_{0}\right)}=m$ and

ii) $\operatorname{Re}\left(z_{0} \frac{W^{\prime \prime}\left(z_{0}\right)}{W^{\prime}\left(z_{0}\right)}\right) \geq m-1$

where $m$ is real and

$$
m \geq k \frac{\left|W\left(z_{0}\right)-a\right|^{2}}{\left|W\left(z_{0}\right)\right|^{2}-|a|^{2}} \geq k \frac{\left|W\left(z_{0}\right)\right|-|a|}{\left|W\left(z_{0}\right)\right|+|a|}
$$

Applying the above lemma $D^{\alpha+p-1} f$ where $f \in M(p)$ we prove the following. 


\section{Theorem}

Let $h(r, s, t) \in H(\alpha)$ and let $f(z) \in M(P)$ satisfy

i) $\left(\frac{D^{\alpha+p} f(z)}{D^{\alpha+p-1} f(z)}, \frac{D^{\alpha+p+1} f(z)}{D^{\alpha+p} f(z)}, \frac{D^{\alpha+p+2} f(z)}{D^{\alpha+p+1} f(z)}\right) \in D \subset C^{3}$ and

ii) $\quad\left|h\left(\frac{D^{\alpha+p} f(z)}{D^{\alpha+p-1} f(z)}, \frac{D^{\alpha+p+1} f(z)}{D^{\alpha+p} f(z)}, \frac{D^{\alpha+p+2} f(z)}{D^{\alpha+p+1} f(z)}\right)\right|<J$

for some $\alpha$ and $J$ such that $\alpha \geq-p+1, \quad J>1$ for all $z \in E_{0}$.

Then we have

$\left|\frac{D^{\alpha+p} f(z)}{D^{\alpha+p-1} f(z)}\right|<J, \quad z \in E_{0}$

\section{Proof}

We define the function $\omega(z)$ in $E_{0}$ by

$$
\frac{D^{\alpha+p} f(z)}{D^{\alpha+p-1} f(z)}=\omega(z) \quad(\alpha \geq-p+1)
$$

for $f(z) \in M(p)$. Then it follows that $\omega(z)$ is either analytic or meromorphic in $E_{0}$ and $\omega(0)=1$ and $\omega(z) \equiv 1$. With the aid of the following easily proved identity

$$
z\left(D^{\alpha+p-1} f(z)\right)^{\prime}=(\alpha+p) D^{\alpha+p} f(z)-(\alpha+2 p) D^{\alpha+p-1} f(z)
$$

We compute

$$
\frac{D^{\alpha+p+1} f(z)}{D^{a+p} f(z)}=\frac{1}{(\alpha+p+1)}\left[1+(\alpha+p) \omega(z)+z \frac{\omega^{\prime}(z)}{\omega(z)}\right] \text { and }
$$




$$
\begin{array}{r}
\frac{D^{\alpha+p+2} f(z)}{D^{\alpha+p+1} f(z)}=\frac{1}{(\alpha+p+2)}\left[2+(\alpha+p) \omega(z)+z \frac{\omega^{\prime}(z)}{\omega(z)}+\right. \\
+(\alpha+p) z \omega^{\prime}(z)+\left[z \omega^{\prime}(z) / \omega(z)\right]+ \\
\left.+\frac{z^{2} \omega^{\prime \prime}(z) / \omega(z)-\left(z \omega^{\prime}(z) / \omega(z)\right)^{2}}{1+(\alpha+p) \omega(z)+z \omega^{\prime}(z) / \omega(z)}\right]
\end{array}
$$

Suppose, if possible, that $z_{0}=r_{0} e^{i \theta}\left(0<r_{0}<1\right)$ and $\left|\omega\left(z_{0}\right)\right|=\max _{|z| \leq r_{0}}|\omega(z)|=J$

Letting $\omega\left(z_{0}\right)=J e^{i \theta}$ and using the lemma with $a=1$ and $k=1$ we get

$$
\begin{aligned}
\frac{D^{\alpha+p+1} f\left(z_{0}\right)}{D^{a+p} f\left(z_{0}\right)} & =\frac{1}{(\alpha+p+1)}\left[1+m+(\alpha+p) m J e^{i \theta}\right] \\
\frac{D^{\alpha+p+2} f\left(z_{0}\right)}{D^{a+p+1} f\left(z_{0}\right)} & =\frac{1}{(\alpha+p+2)} \times \\
& \times\left[2+m+(\alpha+p) m J e^{i \theta}+\frac{m-m^{2}+(\alpha+p) m J e^{i \theta}+L}{1+m+(\alpha+p) J e^{i \theta}}\right]
\end{aligned}
$$

Where $L=z_{0} \omega^{\prime \prime}\left(z_{0}\right) / \omega\left(z_{0}\right)$ and $m \geq(J-1) /(J+1)$.

Further, an application of (ii) in the lemma gives $\operatorname{Re} L \geq m(m-1)$.

Since $h(r, s, t) \in H(\alpha)$, we have

$$
\begin{aligned}
& \left|h\left(\frac{D^{\alpha+p} f\left(z_{0}\right)}{D^{\alpha+p-1} f\left(z_{0}\right)}, \frac{D^{\alpha+p+1} f\left(z_{0}\right)}{D^{\alpha+p} f\left(z_{0}\right)}, \frac{D^{\alpha+p+2} f\left(z_{0}\right)}{D^{\alpha+p+1} f\left(z_{0}\right)}\right)\right|= \\
& \left.\frac{\left(2+m+(\alpha+p) J e^{i \theta}+m-m^{2}+(\alpha+P) m J e^{i \theta}+L\right.}{(\alpha+p+2)\left(1+m+(\alpha+p) J e^{i \theta}\right.}\right) \mid \geq J
\end{aligned}
$$


Which contradicts hypothesis (ii) of the theorem.

Therefore we conclude that

$$
\omega(z)=\left|\frac{D^{a+p} f(z)}{D^{a+p-1} f(z)}\right|<J \text { for all } z \in E_{0}
$$

This proves the theorem.

\section{References}

- A.M.S. Subject Classification (2000) Primary: $30 \mathrm{C} 45$, secondary 34C 40.

1. Lin Jinlin, Note on the Ruscheweyh Derivatives, J. Moth. Anal. Appl., 199, (1996) 936-940.

2. Miller, S.S. and Moconu, P.T., Second order differential inequalities in the complex plan, J. Math. Anal., 65, (1978) 289-305. 